\title{
Development of a predictive model of Crohn's disease proximal small bowel involvement in capsule endos- copy evaluation
}

Authors

Institutions
Eduardo Rodrigues-Pinto ${ }^{1, *}$, Helder Cardoso ${ }^{1, *}$, Bruno Rosa ${ }^{2}$, João Santos-Antunes ${ }^{1}$, Susana Rodrigues ${ }^{1}$, Margarida Marques ${ }^{1}$, Susana Lopes ${ }^{1}$, Andreia Albuquerque ${ }^{1}$, Pedro Carvalho ${ }^{2}$, Maria Moreira ${ }^{2}$, José Cotter ${ }^{2}$, Guilherme Macedo ${ }^{1}$

${ }^{1}$ Gastroenterology Department, Centro Hospitalar São João, Porto, Portugal

${ }^{2}$ Gastroenterology Department, Centro Hospitalar do Alto Ave, Guimarães, Portugal submitted:

5. November 2015 accepted after revision: 11. April 2016

\section{Bibliography}

DOI http://dx.doi.org/

10.1055/s-0042-106961

Published online: 12.5.2016

Endoscopy International Open

2016; 04: E631-E636

(c) Georg Thieme Verlag KG

Stuttgart · New York

E-ISSN 2196-9736

\section{Corresponding author}

\section{Eduardo Rodrigues-Pinto, MD}

Gastroenterology Department Centro Hospitalar São João Porto. Al. Prof. Hernâni Monteiro 4200 - 319 Porto Portugal

Phone: +351919657386 Fax: +351225513601 edu.gil.pinto@gmail.com
Background and study aims: One of the indications for capsule endoscopy (CE) is the detection of proximal small bowel (SB) involvement in Crohn's disease (CD) patients. Our aim was to assess clinical, laboratory and endoscopic predictors associated with proximal SB involvement in $\mathrm{CD}$ patients submitted to $\mathrm{CE}$.

Patients and methods: Retrospective multicenter study in which Lewis score (LS) was systematically determined in $190 \mathrm{CE}$ of patients diagnosed with CD between 2003 and 2014.

Results: Significant inflammatory activity (LS> 135) was present in $23 \%$ of the patients in the first tertile and in $31 \%$ of the patients in the second tertile. Albumin, haemoglobin, and total proteins were significantly lower in patients with a LS> 790 compared to patients with a LS $<135$, while white blood cell counts and C-reactive protein were significantly higher. In the univariable anal-

\section{Introduction \\ $\nabla$}

Crohn's disease (CD) is a chronic inflammatory disorder that can affect all or part of the gastrointestinal tract, with heterogeneous manifestations and adverse events. The exact prevalence of small-bowel (SB) involvement in CD has not been well determined, however, according to Western population-based epidemiologic studies, SB involvement is believed to affect as many as $50 \%$ to $80 \%$ of patients [ $1-3$ ]. The ability to accurately assess the SB is crucial for determining optimal patient management. Until a few years ago, a fluoroscopic examination that included SB followthrough (SBFT) was the suggested technique for SB evaluation [4]. In the past 2 decades, advances in cross-sectional image and capsule endoscopy (CE) have led to widespread use of these noninvasive and well-tolerated approaches [4-6]. One of

\footnotetext{
${ }^{*}$ These authors contributed equally.
}

ysis, a higher risk for proximal SB involvement at $\mathrm{CE}$ was associated with ileal involvement at ileocolonoscopy (OR 2.858, $P=0.006$ ), higher platelets levels (OR 1.005, $P=0.004$ ) and significant weight loss (OR 2.450, $P=0.006$ ). In logistic regression, ileal involvement at ileocolonoscopy (OR 6.817, $P=0.003$ ), stricturing behavior (OR 8.653, $P=0.011$ ) and significant weight loss (OR 3.629, $P=0.028$ ) were independently associated with proximal SB involvement at CE. Considering the ROC curve of this model, a cut-off $>0.249$ predicts proximal SB involvement with $90 \%$ sensitivity and $40 \%$ specificity (AUROC 0.732).

Conclusions: One-third of patients had proximal SB involvement. Predictive factors were significant weight loss, stricturing behaviour, and ileal involvement at ileocolonoscopy. These data help to select $C D$ patients that benefit the most from performing a $\mathrm{CE}$.

the indications for $\mathrm{CE}$ is the detection of proximal involvement of $S B$ in $C D$ patients. The role of $C E$ in patients with known CD has been studied in several trials, which have shown superiority of this test over all other modalities in evaluation of disease activity, severity, and extent of disease, particularly for mild lesions and those located in the proximal SB [7-10]. However, endoscopic findings are nonspecific and must be carefully interpreted within the proper clinical context [11].

Lewis Score (LS) [12] is a cumulative scoring system based on the characteristics and distribution of villous edema, ulceration and stenosis which has been integrated into the latest software from the PillCam ${ }^{\circledR}$ (Given ${ }^{\circledR}$, RAPID Reader ${ }^{\circledR}$ ). It was developed with the purpose of increasing the objectivity and maximizing inter-observer agreement, when assessing SBCE inflammatory activity. It uses a standard terminology for the description of endoscopic lesions, the CE structured terminology (CEST) $[13,14]$, and grades the inflammatory activity through a rank of severity, with the pre- 
mise that the final numerical score reflects the physician's global assessment of disease. With this scoring system, the SB is evaluated according to the division into equal thirds (tertiles) based on the transit time of the capsule. The LS classifies SB inflammatory activity into three grades of severity of inflammation: 1) normal or clinically insignificant mucosal inflammatory change (score < 135 ); 2) mild disease (score $\geq 135<790$ ); and 3) moderate-to-severe disease (score $\geq 790$ ).

There remains considerable uncertainty regarding when to assess disease activity among patients with known CD, as well as it isn't clear how often proximal SB lesions (first and second tertiles) are found. The aim of the current work was to determine frequency and assess clinical, laboratory and endoscopic predictors of proximal SB involvement in patients with established CD submitted to CE.

\section{Patients and methods}

\section{$\nabla$}

Retrospective and multicenter study based on the records of 190 consecutive patients with known CD subjected to SBCE between 2003 and 2014. Patients were excluded if CE was performed as a part of the initial diagnosis of CD. All patients with $C D$ performed patency capsule prior to $\mathrm{CE}$ in order to avoid capsule retention. The diagnosis of CD was made by using widely validated clinical, endoscopic, and histologic criteria [15]. Disease phenotype was determined according to the Montreal classification, based on age at onset, location, and behavior, with perianal and upper gastrointestinal disease as additional modifiers. B1 corresponds to a non-stenosing non-penetrating disease, B2 to a stenosing behaviour, and B3 to a penetrating one. B2 phenotype according to Montreal classification should be defined by the presence of stenosis and clinical symptoms (abdominal pain). Cross-sectional imaging studies were performed in 86 patients prior to CE evaluation (computed tomography enterography [CTE]-45 patients; magnetic resonance enterography [MRE] - 15 patients; SBFT 46 patients). All patients had previous ileocolonoscopy and upper endoscopy. Indication for CE was presence of recurrent abdominal pain, diarrhea, significant weight loss, fever, and/or laboratory findings (anemia, thrombocytosis, hypoalbuminemia, increased inflammatory markers) not explained by findings on upper endoscopy and ileocolonoscopy and/or not resolved by an appropriate pharmacological treatment [16]. Significant weight loss was defined as greater than $5 \%$ of the original weight.

Gender, age, age at onset, disease location, disease behavior, perianal disease, presence of extra-intestinal manifestations, smoking habits, symptoms and laboratory workup at the date of $\mathrm{CE}$ and endoscopic findings closest to the date of CE were thoroughly investigated by reviewing medical records.

To optimize the visualization of the jejunum and ileum with the CE, after an overnight fast, patients ingested $1 \mathrm{~L}$ of PEG 4000 oral solution before capsule ingestion. The endoscopic capsules used were the PillCam SB2 and SB3 (Given Imaging, Yokneam, Israel). PillCam SB2 is a fixed frame rate second-generation capsule (2 frames per second over $8 \mathrm{~h}$ ), while PillCam SB3 is a third-generation capsule with enhanced imaging capabilities with adaptive frame rate. RAPID Reader ${ }^{\circledR}$ was used to review CE images. All capsule registrations previous to RAPID reader availability have been re-read using the new RAPID reader software to calculate the Lewis score. The presence of SB mucosal inflammation on CE was systematically quantified using LS, using the automatic calculator included in the RAPID ${ }^{\circledR}$ software. Normal or clinically insignifi- cant mucosal inflammatory change was defined as LS $<135$, mild inflammation as $135<\mathrm{LS}<790$ and moderate-to-severe disease as $\mathrm{LS} \geq 790$ [12]. In the appropriate clinical setting, the detection of at least 2 ulcers or 1 stricture were considered specific of small bowel involvement by $\mathrm{CD}$. Capsule readers were blinded to endoscopy and CTE/MRE reports.

\section{Statistics}

Categorical variables were described through absolute and relative frequencies and continuous variables were described as mean and standard deviation, median, percentiles, minimum and maximum. Hypotheses were tested about the distribution of continuous variables with non-normal distribution, by using the nonparametric Mann-Whitney and Kruskal-Wallis test, depending on the nature of the hypothesis. Pearson Chi-square and Fisher's exact test were used to test hypotheses about independence of categorical variables, as appropriate.

To identify factors predictive of proximal SB involvement at CE, simple and multivariable analysis was performed using logistic regression. To identify independent predictors of proximal SB involvement at $\mathrm{CE}$, all significant variables evaluated in the univariable analysis (ileal lesions at ileocolonoscopy, significant weight loss, platelets) as well as variables previously associated with a poorer prognosis (age at diagnosis, disease location, disease behaviour) were integrated into a multivariable logistic regression using a stepwise method. The results are shown as odds ratio (OR) with 95\% confidence intervals (CI). With the values obtained, a logistic function was applied to define a predictive model to our outcome. All the reported $P$ values were two-sided, and $P$ values $<0.05$ were considered statistically significant. All data were arranged, processed and analyzed with SPSS ${ }^{\circledR}$ v.20.0 data (Statistical Package for Social Sciences).

\section{Results \\ $\boldsymbol{\nabla}$}

\section{Population}

Baseline demographic characteristics of all 190 patients (108 females (57\%)) with CD are shown in Table 1. At diagnosis, most of the patients $(n=139,75 \%)$ were between 17 and 40 years old, $43 \%(n=81)$ had exclusively ileal involvement (L1) and 75\% ( $n=$ 140) had non-stenosing non-penetrating behavior (B1). Only 16 patients (9\%) had gastroduodenal involvement ( Table 1 ). Twenty percent of the patients had perianal disease. Seventytwo percent of the patients $(n=116)$ had recurrent abdominal pain, 65\% $(n=114)$ had diarrhea, 44\% $(n=75)$ had significant weight loss, $11 \%(n=19)$ had rectal bleeding, and $21 \%(n=25)$ had extra-intestinal manifestations. Twenty-nine patients (20\%) were currently smokers and $12(8 \%)$ were former smokers.

After performance of CE, $43 \%$ of the patients $(n=81)$ started corticosteroids, $17 \%(n=33)$ started azathioprine, $11 \%(n=21)$ started biologic therapy and $6 \%(n=12)$ were submitted to surgery.

\section{Capsule endoscopy \\ $\nabla$}

At the time of CE performance, median hemoglobin was $13.5 \mathrm{~g} / \mathrm{dL}$ (6.9-17.2), median iron was $65 \mu \mathrm{g} / \mathrm{dL}(0-231)$, median ferritin was $23.5 \mathrm{ng} / \mathrm{mL}(0-1727)$, median white blood cell (WBC) counts were $7.5 \times 10^{9}(2.3-23)$, median platelets were $251 \times 10^{9}(72-$ $849)$, median total proteins were $71.6 \mathrm{~g} / \mathrm{dL}(47-84.5)$, median albumin was $42.0 \mathrm{~g} / \mathrm{dL}(22-53.0)$, median erythrocyte sedimenta- 
Table 1 Baseline characteristic of Crohn's disease (CD) patients regarding gender, Montreal classification and smoking habits.

\begin{tabular}{|l|c|}
\hline Characteristics & CD (n=190) \\
\hline Female/male $(\mathrm{n})$ & $108 / 82$ \\
\hline Montreal classification ( $\mathrm{n} ; \%)$ & \\
\hline Age at diagnosis & $19(10 \%)$ \\
\hline A1 & $139(75 \%)$ \\
\hline A2 & $28(15 \%)$ \\
\hline A3 & \\
\hline Disease location & $81(43 \%)$ \\
\hline L1 & $17(9 \%)$ \\
\hline L2 & $73(39 \%)$ \\
\hline L3 & $10(5 \%)$ \\
\hline L1 -4 & $1(1 \%)$ \\
\hline L2 -4 & $5(3 \%)$ \\
\hline L3-4 & $140(75 \%)$ \\
\hline Behaviour & $28(15 \%)$ \\
\hline B1 & $19(10 \%)$ \\
\hline B2 & $24(20 \%)$ \\
\hline B3 & $104(72 \%)$ \\
\hline Perianal disease & $12(8 \%)$ \\
\hline Smoking habits $(n ; \%)$ & $20(20 \%)$ \\
\hline Non-smoker & \\
\hline Ex-smoker & \\
\hline Smoker & \\
\hline
\end{tabular}

tion rate was $11 \mathrm{~mm} / \mathrm{h}(1-89)$ and median C-reactive protein (CRP) was $3.8 \mathrm{mg} / \mathrm{L}(0.2-209.0)$.

The mean LS in the first tertile was $146( \pm 363)$, in the second tertile was $216( \pm 513)$ and in the third tertile was $797( \pm 1042)$. Significant inflammatory activity (LS>135) was present in $23 \%$ of the patients $(n=45)$ in the first tertile, in $31 \%$ of the patients $(n=$ 57 ) in the second tertile, and in $36 \%$ of the patients $(n=68)$ in the first and/or second tertile ( $\bullet$ Table 2 ). Of the 68 patients with LS> 135 in the first and/or second tertile, 45 had ileal involvement at ileocolonoscopy (32 of 45 in the first tertile and 39 of 57 in the second tertile).

There was no correlation between colonic location activity and LS, in neither of the tertiles.

LS was higher in patients with lower hemoglobin levels $(P=0.006)$, higher WBC counts $(P=0.032)$, higher CRPn $(P<0.001)$, lower total protein levels $(P=0.006)$ and lower albumin levels $(P<0.001)$ (- Table 3).
Table 2 Absolute and relative frequencies for the $1^{\text {st }}, 2^{\text {nd }}$ and $3^{\text {rd }}$ tertiles and Lewis score.

\begin{tabular}{|c|c|c|}
\hline Characteristics & \multicolumn{2}{|c|}{$C D(n=190)$} \\
\hline \multicolumn{3}{|c|}{$1^{\text {st }}$ tertile score $(\mathrm{n} ; \%)$} \\
\hline$<135$ & 145 & $76 \%$ \\
\hline $135-790$ & 29 & $15 \%$ \\
\hline$\geq 790$ & 16 & $8 \%$ \\
\hline \multicolumn{3}{|c|}{$2^{\text {nd }}$ tertile score $(n ; \%)$} \\
\hline$<135$ & 131 & $70 \%$ \\
\hline $135-790$ & 37 & $20 \%$ \\
\hline$\geq 790$ & 20 & $11 \%$ \\
\hline \multicolumn{3}{|c|}{$3^{\text {rd }}$ tertile score $(n ; \%)$} \\
\hline$<135$ & 46 & $25 \%$ \\
\hline $135-790$ & 79 & $42 \%$ \\
\hline$\geq 790$ & 61 & $33 \%$ \\
\hline \multicolumn{3}{|l|}{ Lewis score (n; \%) } \\
\hline$<135$ & 38 & $20 \%$ \\
\hline $135-790$ & 81 & $43 \%$ \\
\hline$\geq 790$ & 69 & $37 \%$ \\
\hline
\end{tabular}

Clinical factors associated with proximal small-bowel involvement ( $1^{\text {st }}$ and $2^{\text {nd }}$ tertile) at capsule endoscopy in Crohn's disease patients

In the univariable analysis, a higher risk for proximal SB involvement at $\mathrm{CE}$ was associated with ileal involvement at ileocolonoscopy (OR 2.858, 95\% CI [1.346-6.068], $P=0.006$ ), higher platelets levels (OR 1.005, 95\% CI [1.002-1.009], $P=0.004$ ) and significant weight loss (OR 2.450, 95\% CI [1.293-4.642], $P=0.006$ ) ( $\odot$ Table 4). An older age at diagnosis ( $>40$ years) was associated with a trend toward protection for inflammatory activity at CE (OR 0.3, 95\% CI [0.087-1.039], $P=0.057$ ), while lower albumin levels were associated with a trend toward increased risk for inflammatory activity (OR 0.976, 95\% CI [0.951 - 1.001], $P=0.065$ ). In the multivariable logistic regression, ileal involvement at ileocolonoscopy (OR 6.817, 95\% CI [1.895-24.525], $P=0.003$ ), stricturing behavior (OR 8.653, 95\% CI [1.629-45.972], $P=0.011$ ) and significant weight loss (OR 3.629, 95\% CI [1.138-9.391], $P=$ 0.028 ) were independently associated with proximal SB involvement at CE (AUROC 0.732; 95\% CI [0.648 - 0.817]) (• Fig. 1).

With the aim of simplifying the application of these results on a daily basis so a likelihood of proximal SB involvement could be achieved, a model was created by applying the following logistic function:

$\exp (-2.647+2.158 \times \mathrm{B} 2-0.069 \times \mathrm{B} 3+1.919 \times$ ileal involvement $+1.185 \times$ weight loss $)$ $1+\exp (-2.647+2.158 \times \mathrm{B} 2-0.069 \times \mathrm{B} 3+1.919 \times$ ileal involvement $+1.185 \times$ weight loss $)$

Table 3 Comparisons between Lewis score and laboratory work-up at the date of capsule endoscopy (CE) (hemoglobin, leukocytes, total proteins, albumin, Creactive protein).

\begin{tabular}{|c|c|c|c|c|}
\hline & \multicolumn{3}{|l|}{ Lewis score } & \multirow[t]{2}{*}{$P$ value* } \\
\hline & $<135$ & $135-790$ & $\geq 790$ & \\
\hline Hemoglobin at CE (median; IQR $25-75$ ) & $13.9(13.2-15)$ & $13.5(12.9-14.8)$ & $13.1(11.8-14.3)$ & 0.006 \\
\hline White blood cell counts at CE (median; IQR $25-75$ ) & $6.9(4.9-8.5)$ & $7.2(5.8-9.0)$ & $8.2(6.3-9.7)$ & 0.032 \\
\hline Total proteins at CE (median; $\mathrm{IQR}_{25-75}$ ) & $74.6(71.1-77.2)$ & $70.9(61.2-75.8)$ & $70.8(63.9-73.1)$ & 0.006 \\
\hline Albumin at CE (median; $I^{Q} R_{25-75}$ ) & $45(42.6-47)$ & $41.9(34.8-44.0)$ & $41(37.7-43.0)$ & $<0.001$ \\
\hline C-reactive protein at CE (median; ${ } \mathrm{QR}_{25-75}$ ) & $3.4(1.0-6.1)$ & $2.8(1.2-6.3)$ & $7.65(3.1-16.9)$ & $<0.001$ \\
\hline
\end{tabular}

${ }^{*}$ Kruskall-wallis test 
Table 4 Clinical factors associated with proximal small-bowel involvement at capsule endoscopy in Crohn's disease patients.

\begin{tabular}{|c|c|c|c|c|c|c|}
\hline & \multicolumn{3}{|c|}{ Univariate analysis } & \multicolumn{3}{|c|}{ Multivariate analysis ${ }^{1}$} \\
\hline & $\mathrm{OR}^{2}$ & $95 \% \mathrm{Cl}$ & $P$ value ${ }^{+}$ & $\mathrm{OR}^{\&}$ & $95 \% \mathrm{Cl}$ & $P$ value $^{3}$ \\
\hline \multicolumn{7}{|c|}{ Age at diagnosis (years-old) } \\
\hline$\leq 17$ & Ref. & & & & & \\
\hline $17-40$ & 0.522 & $0.199-1.368$ & 0.186 & & & \\
\hline$>40$ & 0.300 & $0.087-1.039$ & 0.057 & & & \\
\hline \multicolumn{7}{|l|}{ Disease location } \\
\hline $\mathrm{L} 1+\mathrm{L} 1 \mathrm{~L} 4$ & Ref. & & & & & \\
\hline $\mathrm{L} 2+\mathrm{L} 2 \mathrm{~L} 4$ & 0.184 & $0.022-1.545$ & 0.119 & & & \\
\hline $\mathrm{L} 3+\mathrm{L} 3 \mathrm{~L} 4$ & 1.416 & $0.668-3.004$ & 0.364 & & & \\
\hline \multicolumn{7}{|l|}{ Disease behavior } \\
\hline B1 & Ref. & & & Ref. & & \\
\hline B2 & 1.560 & $0.688-3.539$ & 0.287 & 8.653 & $1.629-45.972$ & 0.011 \\
\hline B3 & 0.831 & $0.294-2.321$ & 0.724 & 0.933 & $0.250-4.252$ & 0.929 \\
\hline \multicolumn{7}{|c|}{ Ileal involvement at ileocolonoscopy } \\
\hline No & Ref. & & & Ref. & & \\
\hline Yes & 2.858 & $1.346-6.068$ & 0.006 & 6.817 & $1.895-24.525$ & 0.003 \\
\hline Hemoglobin & 0.891 & $0.740-1.073$ & 0.224 & & & \\
\hline C-reactive protein & 1.020 & $0.997-1.045$ & 0.094 & & & \\
\hline Platelets & 1.005 & $1.002-1.009$ & 0.004 & & & \\
\hline Total proteins & 0.990 & $0.974-1.007$ & 0.258 & & & \\
\hline Albumin & 0.976 & $0.951-1.001$ & 0.065 & & & \\
\hline \multicolumn{7}{|l|}{ Weight loss } \\
\hline No & Ref. & & & Ref. & & \\
\hline Yes & 2.450 & $1.293-4.642$ & 0.006 & 3.629 & $1.138-9.391$ & 0.028 \\
\hline \multicolumn{7}{|l|}{ Abdominal pain } \\
\hline No & Ref. & & & & & \\
\hline Yes & 1.130 & $0.547-2.332$ & 0.741 & & & \\
\hline \multicolumn{7}{|l|}{ Diarrhea } \\
\hline No & Ref. & & & & & \\
\hline Yes & 1.751 & $0.893-3.433$ & 0.103 & & & \\
\hline \multicolumn{7}{|c|}{ Extra-intestinal manifestations } \\
\hline No & Ref. & & & & & \\
\hline Yes & 0.688 & $0.270-1.750$ & 0.432 & & & \\
\hline \multicolumn{7}{|l|}{ Perianal disease } \\
\hline No & Ref. & & & & & \\
\hline Yes & 0.851 & $0.342-2.117$ & 0.729 & & & \\
\hline
\end{tabular}

1 independent variables: age at diagnosis, disease location, disease behaviour, ileal lesions at ileocolonoscopy, weight loss, platelets

${ }^{2} \mathrm{OR}$ - odds ratio

${ }^{3} P$ value: overall comparison between groups

Assuming a sensitivity of $90 \%$ and a specificity of $40 \%$, we consider the cut-off of 0.249 as clinically relevant, with a positive predictive value (PPV) of $61 \%$, a negative predictive value (NPV) of $76 \%$ and an accuracy of $70 \%$, regarding likelihood of small bowel involvement.

\section{Discussion \\ $\nabla$}

Our purpose was to determine which clinical, laboratory and endoscopic factors predict proximal SB involvement in CD patients submitted to $\mathrm{CE}$. One of the indications of $\mathrm{CE}$ is the assessment of disease activity and extent in patients with known CD. However, most of previous studies are related to the use of $\mathrm{CE}$ in suspected CD with previous negative ileocolonoscopy [16-20]. CE findings suggestive of $C D$ involvement can be rather nonspecific and include ulceration, erythema, mucosal oedema and strictures. This presents a significant challenge to the interpreting physician because minor mucosal breaks may occur in $10 \%$ to $15 \%$ of normal individuals while mucosal erosions are present in two-thirds of patients taking nonsteroidal anti-inflammatory drugs [21]. This could explain the variability of the "diagnostic yield" of CE. Also, it must be considered that the "diagnostic yield" is different to either "sensitivity" or "specificity" [22]. Yield of $C E$ varies depending on the type of patient, being lower when performed in patients with only abdominal pain compared with patients with abdominal pain and diarrhea. LS, an inflammation score that is incorporated in Given Imaging Software, is likely to enhance our ability to assess established CD at CE [14].

The largest comparative study of multiple SB imaging modalities involved a comparison of CE, CTE, and MRE performed after ileocolonoscopy. The results reported a significantly superior detection of CD in the proximal SB by CE compared with both CTE and MRE. In suspected or newly diagnosed $C D$, MRE and CTE have comparable sensitivities and specificities and, in patients without endoscopic or clinical suspicion of stenosis, CE would be the firstline modality for detection of SB CD beyond the reach of the colonoscope. Overall, these comparative studies suggest that $\mathrm{CE}$ is more sensitive than SBFT and may be more sensitive than crosssectional imaging. Several meta-analyses $[9,10,23,24]$ showed 


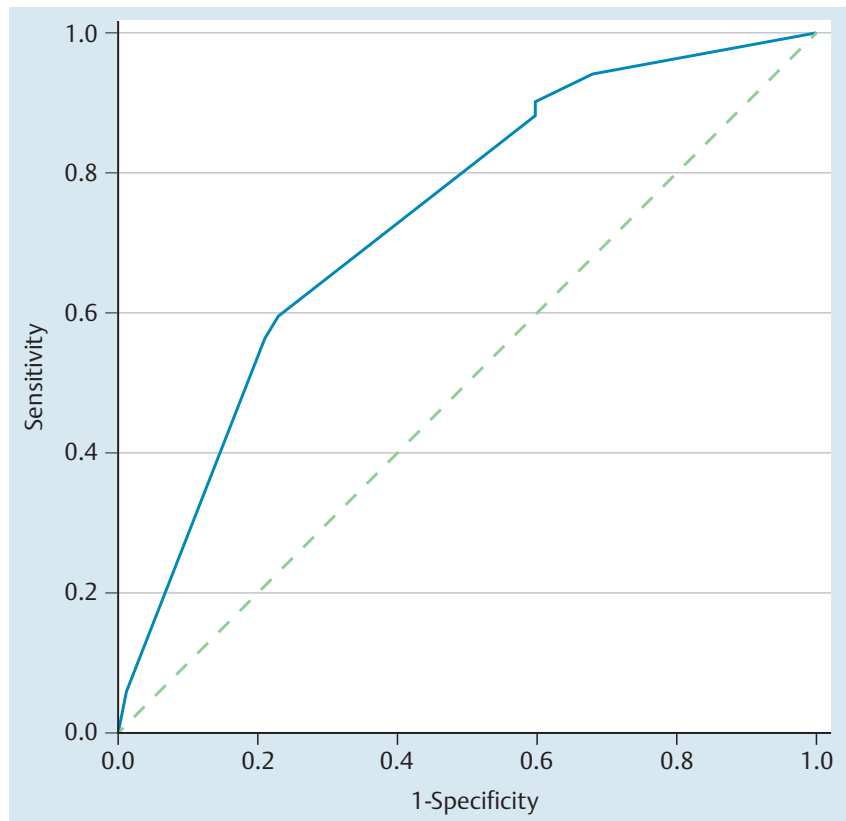

Diagonal segments are produced by ties.

Fig. 1 ROC curve for proximal SB involvement considering CD behavior, ileal involvement at ileocolonoscopy, and weight loss. ROC, Receiver operating characteristic; SB, small-bowel; CD, Crohn's disease

that CE has superior diagnostic yields to SB barium studies, ileocolonoscopy, push enteroscopy and CTE in both suspected and established SB CD. CE appears to be better than MRE at identifying SB mucosal lesions, while MRE is more accurate at diagnosing mural, peri-mural and extra-enteric manifestations $[25,26]$.

Approximately $50 \%$ of patients with symptomatic ileal and/or colonic $\mathrm{CD}$ have their proximal SB affected, with most common distributions including the proximal ileum (67\%), jejunum (53\%) and duodenum (32\%) [27]. Recent studies show that proximal involvement is associated with younger age, non-smoking status, co-existence of ileal involvement at ileocolonoscopy, and stenosing pattern $[28,29]$.

As proposed by the first consensus of the International Conference on Capsule Endoscopy (ICCE), ileocolonoscopy must always be performed prior to $\mathrm{CE}$, considering it for $\mathrm{CD}$ diagnosis or exclusion if the patient presents suspicious symptoms (abdominal pain or persistent diarrhea) as well as extraintestinal manifestations, alteration of inflammatory markers, or abnormalities in other imaging tests [30]. The role of CE in patients with established CD has mainly been studied to explain new symptoms and to demonstrate mucosal healing, with no previous reports in the literature regarding predictive factors of SB involvement and which $\mathrm{CD}$ patients benefit the most from performing a $\mathrm{CE}$ at diagnosis. In this large cohort, it was possible to show that SBCE identifies proximal lesions (first and second tertiles) in $36 \%$ of patients $(n=68)$ with established $C D$, when only $9 \%$ were considered to have gastroduodenal involvement at baseline. Even though $34 \%$ of the patients $(n=65)$ were already known to have poor prognostic factors at baseline (gastroduodenal involvement, perianal disease or extraintestinal manifestations), despite of already being on immunomodulators or biologic therapy at the time of CE performance, they persisted with unexplained symptoms or abnormal laboratory findings. Of the 68 patients with proximal lesions in the SB, 23 did not have ileal involvement at ileocolonoscopy. LS was higher in patients with lower hemoglo- bin levels, higher WB counts, higher CRP, lower total protein levels, and lower albumin levels.

In the multivariable analysis, significant weight loss, stricturing behavior and ileal involvement at ileocolonoscopy were predictive factors of $\mathrm{SB}$ involvement. Considering the ROC curve of the model, a cut-off higher than 0.249 predicts proximal SB involvement with $90 \%$ sensitivity and $40 \%$ specificity. Even though the specificity of the ROC model is low, it has a high sensitivity and a moderately high NPV (76\%). This cut-off helps to select which patients may benefit the most from performing $\mathrm{CE}$, once it would detect $90 \%$ of the patients with lesions in the proximal SB, inaccessible to conventional endoscopy/ileocolonoscopy; Likewise, patients with a cut-off lower than 0.249 are less likely to benefit from performing $\mathrm{CE}$, once $76 \%$ of them won't have proximal $\mathrm{SB}$ involvement. CE should be considered when a change in disease management is foreseen [22]. As previously reported [29], the prevalence of jejunal lesions is higher when the terminal ileum is involved and associated with an increased risk of further clinical relapse; it may be regarded as a factor of severity. Our results reinforce this point. Furthermore, given $\mathrm{CE}$ high diagnostic yield for established disease, its findings may influence management changes and clinical monitoring. It is well known that the presence of SB involvement is associated with an increased risk of further clinical relapse [28] and therefore an early and rapid diagnosis is necessary. Therefore, in the presence of symptoms or signs unexplained by previous exams, particularly significant weight loss, stricturing behavior or ileal involvement at colonoscopy, CE may detect lesions accounting for manifestations beyond the duodenum and terminal ileum, otherwise inaccessible to conventional endoscopy. Once $\mathrm{CE}$ is superior to cross sectional imaging for the detection of proximal lesions, CE could be a better exam to be performed, in patients with unexplained symptoms and a location of the disease in the terminal ileum previously seen at ileocolonoscopy. Even though the findings obtained by our model are of undoubted interest, they require validation, in order to help selecting the CD patients that benefit the most from performing a $\mathrm{CE}$ at diagnosis.

\section{Competing interests: None}

\section{References}

1 Annese V, Daperno M, Rutter MD et al. European Crohn's and Colitis Organisation. European evidence based consensus for endoscopy in inflammatory bowel disease. J Crohns Colitis 2013; 7: 982 - 1018

2 Gower-Rousseau C, Vasseur F, Fumery M et al. Epidemiology of inflammatory bowel diseases: new insights from a French population-based registry (EPIMAD). Dig Liver Dis 2013; 45: 89-94

3 Nuij VJ, Zelinkova Z, Rijk MC et al. Dutch Delta IBD Group. Phenotype of inflammatory bowel disease at diagnosis in the Netherlands: a population-based inception cohort study (the Delta Cohort). Inflamm Bowel Dis 2013; 19: 2215-2222

4 Stuart S, Conner T, Ahmed A et al. The smaller bowel: imaging the small bowel in paediatric Crohn's disease. Postgrad Med J 2011; 87: 288 297

5 Di Nardo G, Aloi M, Oliva $S$ et al. Investigation of small bowel in pediatric Crohn's disease. Inflamm Bowel Dis 2012; 18: 1760 - 1776

6 Fletcher JG, Fidler JL, Bruining DH et al. New concepts in intestinal imaging for inflammatory bowel diseases. Gastroenterology 2011; 140: $1795-1806$

7 Leighton JA, Legnani P, Seidman EG. Role of capsule endoscopy in inflammatory bowel disease: where we are and where we are going. Inflamm Bowel Dis 2007; 13: 331 - 337

8 Marmo R, Rotondano G, Piscopo R et al. Meta-analysis: capsule enteroscopy vs. conventional modalities in diagnosis of small bowel diseases. Aliment Pharmacol Ther 2005; 22: 595-604 
9 Triester SL, Leighton JA, Leontiadis GI et al. A meta-analysis of the yield of capsule endoscopy compared to other diagnostic modalities in patients with non-stricturing small bowel Crohn's disease. Am J Gastroenterol 2006; 101: 954-964

10 Dionisio PM, Gurudu SR, Leighton JA et al. Capsule endoscopy has a significantly higher diagnostic yield in patients with suspected and established small-bowel Crohn's disease: a meta-analysis. Am J Gastroenterol 2010; 105: 1240-1248; quiz 1249

11 Solem CA, Loftus EVJr, Fletcher JG et al. Small-bowel imaging in Crohn's disease: a prospective, blinded, 4-way comparison trial. Gastrointest Endosc 2008; 68: 255 - 266

12 Gralnek IM, Defranchis R, Seidman E et al. Development of a capsule endoscopy scoring index for small bowel mucosal inflammatory change. Aliment Pharmacol Ther 2008; 27: 146-154

13 Korman LY. Standard terminology for capsule endoscopy. Gastrointest Endosc Clin N Am 2004; 14: 33-41

14 Korman $L Y$, Delvaux M, Gay G et al. Capsule endoscopy structured terminology (CEST): proposal of a standardized and structured terminology for reporting capsule endoscopy procedures. Endoscopy 2005; 37 : 951-959

15 Dignass A, Van Assche G, Lindsay JO et al. European Crohn's and Colitis Organisation (ECCO). The second European evidence-based Consensus on the diagnosis and management of Crohn's disease: Current management. J Crohns Colitis 2010; 4: 28-62

16 Valle J, Alcántara M, Pérez-Grueso MJ et al. Clinical features of patients with negative results from traditional diagnostic work-up and Crohn's disease findings from capsule endoscopy. J Clin Gastroenterol 2006; 40: $692-696$

17 Herrerías JM, Caunedo A, Rodríguez-Téllez M et al. Capsule endoscopy in patients with suspected Crohn's disease and negative endoscopy. Endoscopy 2003; 35: 564-568

18 Fireman Z, Mahajna E, Broide E et al. Diagnosing small bowel Crohn's disease with wireless capsule endoscopy. Gut 2003; 52: 390-392

19 Girelli CM, Porta P, Malacrida Vet al. Clinical outcome of patients examined by capsule endoscopy for suspected small bowel Crohn's disease. Dig Liver Dis 2007; 39: 148-154
20 Fireman Z, Eliakim R, Adler S et al. Capsule endoscopy in real life: a fourcentre experience of 160 consecutive patients in Israel. Eur J Gastroenterol Hepatol 2004; 16: 927-931

21 Sidhu R, Brunt $L K$, Morley SR et al. Undisclosed use of nonsteroidal antiinflammatory drugs may underlie small-bowel injury observed by capsule endoscopy. Clin Gastroenterol Hepatol 2010; 8: 992-995

22 Bourreille A, Ignjatovic A, Aabakken L et al. World Organisation of Digestive Endoscopy (OMED) and the European Crohn's and Colitis Organisation (ECCO). Role of small-bowel endoscopy in the management of patients with inflammatory bowel disease: an international OMEDECCO consensus . Endoscopy 2009; 41: 618-637

23 Pasha SF, Leighton JA, Das A et al. Double-balloon enteroscopy and capsule endoscopy have comparable diagnostic yield in small-bowel disease: a meta-analysis. Clin Gastroenterol Hepatol 2008; 6: 671-676

24 Chen X, Ran ZH, Tong JL. A meta-analysis of the yield of capsule endoscopy compared to double-balloon enteroscopy in patients with small bowel diseases. World J Gastroenterol 2007; 13: 4372 - 4378

25 Crook DW, Knuesel PR, Froehlich JM et al. Comparison of magnetic resonance enterography and video capsule endoscopy in evaluating small bowel disease. Eur J Gastroenterol Hepatol 2009; 21: 54-65

26 Tillack C, Seiderer J, Brand S et al. Correlation of magnetic resonance enteroclysis (MRE) and wireless capsule endoscopy (CE) in the diagnosis of small bowel lesions in Crohn's disease. Inflamm Bowel Dis 2008; 14: $1219-1928$

27 Petruzziello C, Onali S, Calabrese E et al. Wireless capsule endoscopy and proximal small bowel lesions in Crohn's disease. World J Gastroenterol 2010; 16: 3299-3304

28 Lazarev M, Huang C, Bitton A et al. Relationship between proximal Crohn's disease location and disease behavior and surgery: a crosssectional study of the IBD Genetics Consortium. Am J Gastroenterol 2013; 108: 106-112

29 Flamant $M$, Trang $C$, Maillard $O$ et al. The prevalence and outcome of jejunal lesions visualized by small bowel capsule endoscopy in Crohn's disease. Inflamm Bowel Dis 2013; 19: 1390-1396

30 Lewis BS. Expanding role of capsule endoscopy in inflammatory bowel disease. World J Gastroenterol 2008; 14: 4137-4141 